Revue d'histoire de l'Amérique française

BEVUE D.HISTOIRE DE L'AMÉRIQUE FRANÇAISE

\title{
Massell, David, Quebec Hydropolitics. The Peribonka Concessions of the Second World War (Montréal et Kingston, McGill-Queen's University Press, 2011), 242 pages
}

\section{Claude Bellavance}

Volume 66, numéro 3-4, hiver-printemps 2013

URI : https://id.erudit.org/iderudit/1025548ar

DOI : https://doi.org/10.7202/1025548ar

Aller au sommaire du numéro

Éditeur(s)

Institut d'histoire de l'Amérique française

ISSN

0035-2357 (imprimé)

1492-1383 (numérique)

Découvrir la revue

Citer ce compte rendu

Bellavance, C. (2013). Compte rendu de [Massell, David, Quebec Hydropolitics.

The Peribonka Concessions of the Second World War (Montréal et Kingston,

McGill-Queen's University Press, 2011), 242 pages]. Revue d'histoire de

l'Amérique française, 66(3-4), 484-487. https://doi.org/10.7202/1025548ar d'utilisation que vous pouvez consulter en ligne.

https://apropos.erudit.org/fr/usagers/politique-dutilisation/ 
compositeurs. Elle lève ainsi le voile sur une période vraiment trop peu étudiée des lettres québécoises et montre l'ampleur des recherches qu’il reste à poursuivre.

LUCIE ROBERT

Centre de recherche interuniversitaire sur la littérature et la culture québécoises (CRILCQ)

Département d'études littéraires Université du Québec à Montréal

Massell, David, Quebec Hydropolitics. The Peribonka Concessions of the Second World War (Montréal et Kingston, McGill-Queen's University Press, 2011), 242 pages.

David Massell avait déjà publié en 2000 chez le même éditeur un premier ouvrage consacré au développement des ressources hydroélectriques de la région Saguenay-Lac-Saint-Jean au début du $\mathrm{XX}^{\mathrm{e}}$ siècle. Adoptant la même approche, il aborde cette fois-ci une autre période clé de l'histoire de l'hydroélectricité au Québec, celle de la Seconde Guerre mondiale. Si les projets d'Alcan sont au cœur de l'ouvrage, le travail des ingénieurs et fonctionnaires de l'État québécois est aussi examiné, de même que la politique énergétique du gouvernement québécois, principalement lors du mandat d'Adélard Godbout. En raison de la guerre et de la mobilisation exceptionnelle des ressources qu'elle entraîne, l'État fédéral joue également un rôle significatif dans l'établissement des priorités et des calendriers. Enfin, Massell introduit dans cette deuxième étude un point de vue nouveau, celui des Innus (Montagnais), la communauté autochtone qui fréquente depuis plusieurs générations le bassin hydrographique de la Péribonka, le principal affluent du Saguenay.

L'ouvrage comporte six courts chapitres et une longue conclusion. Ils forment le récit des événements menant à la création de réservoirs à la tête des rivières Manouane et Péribonka (ouvrages visant à augmenter la puissance installée des centrales hydroélectriques d'Alcan dans la région), de même qu'à la mise en chantier de la centrale Shipshaw. Avant d'être convoité par le grand capital, les bassins hydrographiques de ces deux cours d'eau étaient le territoire de chasse des Innus. Puisant dans un matériel largement inédit - des enquêtes orales menées auprès des chasseurs-trappeurs montagnais par des membres de la communauté supervisés par l'anthropologue Denis Brassard à la fin des années 1970 et 
quelques entretiens qu'il a lui-même récemment réalisés -, Massell nous fait d'abord découvrir l'espace vécu de ces populations encore à deminomades durant les années 1930.

Ce bref essai de "préhistoire» industrielle du bassin versant de la Péribonka montre à quel point leur connaissance concrète du territoire a longtemps surclassé celle qu'en avaient les ingénieurs de l'État et des grandes entreprises. À partir des années 1910, toutefois, ces derniers vont montrer un intérêt croissant pour les potentiels du Saguenay et de ses tributaires, multipliant les études hydrauliques et des travaux d'exploration sur le terrain. Les premiers ouvrages hydroélectriques majeurs au Saguenay sont mis en chantier par Duke-Price et Alcoa au cours de la décennie suivante, alors qu'on réalise une première cartographie précise des bassins versants du Nord en mettant à profit une nouvelle source, les photographies aériennes. Les années 1930 marquent toutefois un temps d'arrêt des grands projets. La communauté innue peut ainsi continuer à fréquenter pour un temps ses territoires de chasse et de pêche traditionnels où les familles de chasseurs passent encore la majeure partie de l'année.

La forte demande d'aluminium pendant la Deuxième Guerre mondiale relance les projets d'Alcan (filiale canadienne d'Alcoa) dans la région. La production massive d'avions, et par conséquent d'aluminium, est alors considérée par les Alliés comme l'une des conditions nécessaires pour vaincre l'Allemagne nazie. L'étude de Massell montre bien le rôle crucial joué par les services canadiens et américains de coordination de l'industrie en temps de guerre. Les émissaires des gouvernements Roosevelt et Mackenzie King font tout en leur pouvoir pour accroître rapidement l'offre d'énergie au Canada; le développement des potentiels encore non exploités du Saguenay paraît alors l'une des meilleures façons d'y parvenir. Aussi, non seulement appuient-ils financièrement Alcan, mais ils interviennent auprès du gouvernement du Québec pour qu'il cède à celle-ci les droits d'exploitation encore non aliénés.

Rappelons que la question de l'électricité au Québec est considérée depuis au moins une décennie comme une question d'intérêt national. On y voit, en effet, à la fois une illustration de la dépendance économique de la majorité canadienne-française et un moyen de favoriser son émancipation. Plusieurs intellectuels et activistes critiquent durement le Trust de l'électricité de même que les gouvernements qui, depuis le début du siècle, ont cédé l'énergie des cours d'eau à très bas prix. En 1939 , tous les partis politiques ont mis à l'ordre du jour la nationalisation 
du secteur de l'électricité ou, à tout le moins, la création d'une grande entreprise publique chargée de faire concurrence au secteur privé. La question de l'électricité est d'ailleurs particulièrement délicate dans la région du Saguenay-Lac-Saint-Jean où Alcoa et ses associés ont géré le niveau des réservoirs sans tenir compte des droits des populations riveraines, inondant les localités au pourtour du lac Saint-Jean.

Tout comme dans son premier livre, Massell nous fait découvrir des facettes généralement méconnues du développement d'une expertise technique au sein de l'appareil d’État québécois. Il s'agit, à notre avis, de sa contribution la plus précieuse et la plus originale. Le rôle majeur joué par les Latreille, Pigeon, McCammon, Normandin et autres experts au service des divers organismes de régulation de l'État québécois pour éviter l'aliénation à rabais des ressources du domaine public est analysé avec méthode et perspicacité. On constate notamment que, si le gouvernement Godbout a accepté plusieurs des demandes pressantes des représentants d'Alcan et du gouvernement fédéral - allant même jusqu'à tolérer que l'entreprise d'aluminium procède aux travaux menant à la création d'un immense réservoir à la tête de la Péribonka plusieurs mois avant l'émission de l'ordre en conseil régularisant la situation -, il a néanmoins refusé de concéder le potentiel des bassins voisins de la Côte-Nord (Bersimis et Outardes), les réservant à Hydro-Québec qui allait voir le jour quelques mois plus tard. Ce choix stratégique sera d'ailleurs reconduit par l'État québécois après l'élection du deuxième gouvernement de Maurice Duplessis en 1944.

L'auteur nous livre également une réflexion tout à fait intéressante sur le contexte géopolitique et économique continental dans lequel évoluent Alcan et Alcoa pendant la guerre et sur leur capacité à tirer profit de cette période d'exception pour renforcer leur position stratégique, grâce notamment à la prise de contrôle de gigantesques ressources hydroélectriques. Massell conclut son ouvrage en tentant de lier la réalisation des projets d'Alcan au Saguenay-Lac-Saint-Jean à la première nationalisation de 1944. Il estime qu'après la construction de Shipshaw, le gouvernement Godbout aurait été contraint de jeter du lest face à une opinion publique manipulée par Duplessis et ses alliés, bref que les développements hydroélectriques au Saguenay auraient été une sorte de déclencheur menant à la création d'Hydro-Québec. L'hypothèse est amenée avec prudence et d'autres éléments sont pris en considération comme le dépôt des études 
de la Régie de l'électricité sur les pratiques financières de Montreal Light Heat and Power.

Le tableau de la situation au sortir de la guerre demeure néanmoins trop incomplet pour pouvoir souscrire pleinement à l'interprétation de l'auteur. À notre avis, pour aller plus loin, il faudrait examiner les stratégies d'autres joueurs tout en élargissant l'angle d'approche afin d'y intégrer certaines perspectives analytiques propres à l'histoire des techniques de même qu'à l'histoire des grandes entreprises et de l'État. Quoi qu'il en soit, il faut saluer la parution de ce livre bien construit et documenté (signalons que l'auteur a réalisé son étude sans avoir pu hélas avoir accès aux archives d'Alcan et que c'est donc du côté des sources gouvernementales qu'il a puisé l'essentiel de son matériel original), souvent passionnant, qui permet de mieux comprendre une période cruciale de la formation du Québec contemporain.

Claude Bellavance CIEQ et Département des sciences humaines Université du Québec à Trois-Rivières

Théorêt, Hugues, Les chemises bleues: Adrien Arcand, journaliste antisémite canadien-français (Québec, Septentrion, 2012), 410 p.

Écrire sur Adrien Arcand peut s'avérer un exercice périlleux. Près de cinquante ans après sa mort, ce dernier continue de faire couler beaucoup d'encre en raison de ses idées et de la vision du monde qu'elles sous-tendent. Journaliste antisémite, anticommuniste et profondément catholique, Arcand demeure une figure controversée identifiée au Parti national social chrétien (PNSC) qu'il établit au Québec durant l'entredeux-guerres. Toutefois, ce serait une erreur que de limiter son étude à cette simple dimension.

Fruit d'une recherche documentaire minutieuse, l'ouvrage d'Hugues Théorêt permet de mieux comprendre ce personnage à la lumière de son époque, de ses écrits, de son réseau de relations et de connaissances. Mariant l'approche biographique à l'histoire intellectuelle, l'auteur fait œuvre utile en rattachant Arcand à un courant antisémite mondial. À travers sa correspondance, il esquisse les contours de la diffusion de ses publications hors des frontières et expose les liens unissant Arcand avec 